\title{
Expression and clinical significance of IncRNA TCL6 in serum of patients with preeclampsia
}

\author{
HONG WANG, GUIMEI SHEN, MENGSI LIU, LINJUAN MAO and HUI MAO \\ Department of Obstetrics and Reproductive Center, \\ The Affiliated Hospital of Yunnan University, Kunming, Yunnan 650000, P.R. China
}

Received November 25, 2020; Accepted May 7, 2021

DOI: 10.3892/etm.2021.10963

\begin{abstract}
Preeclampsia is a common syndrome in pregnancy and a leading cause of mortality of pregnant females and their infants. To investigate the diagnostic and prognostic utility of lncRNA T-cell leukemia/lymphoma 6 (TCL6) in patients with preeclampsia, 120 singleton pregnant females with preeclampsia and another 100 healthy pregnant control subjects were analyzed in the present study. The expression of IncRNA TCL6 in the serum of the included patients was detected. Receiver operating characteristic curve analysis was applied to evaluate the efficiency of lncRNA TCL6 in terms of preeclampsia diagnosis and grading. Kaplan-Meier analysis was adopted to assess the effect of lncRNA TCL6 expression on the rate of adverse pregnancy. Multivariate logistic regression was used to determine high-risk factors of adverse pregnancy. The results indicated that lncRNA TCL6 was significantly increased in the serum of patients with preeclampsia. Furthermore, TCL6 was elevated in subgroups of patients with early-onset or severe preeclampsia and with Haemolysis, Elevated Liver enzymes and Low Platelet count syndrome in comparison with other patients with preeclampsia. High expression of TCL6 in pregnant females corresponded to a higher rate of adverse pregnancy outcomes. Severe preeclampsia, early-onset preeclampsia and high TCL6 expression were identified as independent risk factors for adverse pregnancy outcomes. For each unit increase in TCL6 expression, a 9.5-fold increase of the risk of adverse maternal and fetal outcomes was determined. Collectively, high expression of lncRNA TCL6 may assist the diagnosis and grading of preeclampsia and may be adopted as an independent risk factor for adverse pregnancy outcomes.
\end{abstract}

Correspondence to: Mr. Hui Mao, Department of Obstetrics and Reproductive Center, The Affiliated Hospital of Yunnan University, 176 Qingnian Road, Wuhua, Kunming, Yunnan 650000, P.R. China E-mail: huim0701@163.com

Key words: preeclampsia, long non-coding RNA, T-cell leukemia/lymphoma 6 , adverse pregnancy, diagnosis, prognosis, HELLP syndrome, receiver operating characteristic curve, logistic regression

\section{Introduction}

Preeclampsia is a common health condition in pregnant females, which is characterized by hypertension and multiple organ failure including renal, liver and lung dysfunction (1). It is a leading cause of mortality of pregnant females and infants (2). Preeclampsia poses a risk to millions of pregnant females during pregnancy, among which almost 300,000 females decease along the process and $>500,000$ infants expire annually worldwide, including the death prior to birth and afterwards (3). According to the severity parameters of preeclampsia, pregnant females with signs of blood pressure $\geq 160 / 110 \mathrm{mmHg}$, thrombocytopenia [with platelet count (PLT) $<100 \times 10^{9} / 1$ ], renal insufficiency (serum creatinine two times higher than the normal limit) and serum alanine aminotransferase (ALT) more than two times of the normal limit, are at a higher risk of severe preeclampsia (SPE) (4). Haemolysis, Elevated Liver enzymes and Low Platelet count (HELLP) syndrome is a high-risk factor for mortality of pregnant females and infants characterized by signs of haemolysis, elevation of the liver enzymes and low PLT (5). HELLP syndrome is a serious complication of pregnancy owing to hypertensive disorder, which is a cause of preeclampsia, accounting for about $1 / 4$ of preeclampsia (5). In the past years, researchers have been concentrating on the cause and the pathological mechanism of preeclampsia (6). Extensive research is necessary for the adoption of epidemiological methods to follow up the prognosis of patients with preeclampsia and determine risk factors to provide evidence-based medical insight into assessing the condition and preventing adverse outcomes of pregnant females and their infants.

Long noncoding RNAs (lncRNAs) are a class of non-translatable RNA longer than 200 nucleotides; stable in serum and other biological fluids, they function as a definitive indicator for evaluating disease and tissue specificity (7). Since the initial discovery of IncRNAs, accumulating evidence has indicated their significance in the development and functioning of the placenta, where anomalies in placental differentiation and function critically affect the pregnancy outcome and postpartum maternal and infant health (8). T-cell leukemia/lymphoma 6 (TCL6) is an lncRNA identified in T-cell leukemia; it is located in the breakpoint cluster region on chromosome 14q32, also known as TNG1 or TNG2 (9). Emerging evidence has supported the potential role of TCL6 
as a tumor suppressor (10-12). A study identified an association between IncRNA TCL6 and immune infiltration and suggested it may be adopted as a molecular marker for the prognosis of breast cancer (13). A recent study indicated that TCL6 is highly expressed in the placenta of patients with threatened abortion and may serve as a modulator of trophoblast function via the epidermal growth factor receptor signaling pathway (14). In addition, a previous study has elucidated the involvement of TCL6 in the pathogenesis of preeclampsia, where its high expression may inhibit the proliferation of trophoblast cells (15).

Currently, only a limited number of studies have focused on TCL6 and preeclampsia and the relationship between the expression of IncRNA TCL6 and the diagnosis, grading and prognosis of preeclampsia warrants further exploration. The aim of the present study was to investigate the lncRNA TCL6 expression in the serum of pregnant females with preeclampsia as compared with that in normal controls and assess the association of TCL6 with the diagnosis and severity of preeclampsia and pregnancy outcomes.

\section{Materials and methods}

Study subjects. Pregnant women from January 2018 to December 2019 in obstetrics department of The Affiliated Hospital of Yunnan University were selected. The purpose, operation process and possible impact of the clinical study were explained to them through interview. The case data were reviewed by the medical professionals to determine whether they were suitable for the study. The patients who were fully informed and agreed to participate in the study were included in the study. A total of 120 females during late singleton pregnancy (gestational age $\geq 28$ weeks) with preeclampsia hospitalized at the Second People's Hospital of Yunnan Province (Kunming, China) between January 2018 and December 2019 were enrolled as the experimental group of the present study, including 60 cases of mild preeclampsia (MPE) and 60 cases of SPE. Another 100 healthy females in late singleton pregnancy were enrolled as the control group. Healthy subjects during the same period were matched with preeclampsia patients according to age \pm 3 years and gestational age \pm 2 weeks. All patients were admitted to the hospital when they were enrolled until delivery and discharge. From all participants, whole-blood samples and 24-h urine samples were collected. The diagnosis of preeclampsia and grouping of subjects were performed strictly according to the hypertension in pregnancy published by the American College of Obstetricians and Gynecologists in 2013 (16) and the Diagnosis and treatment guidelines of hypertensive disorders in pregnancy published by the Obstetrics and Gynecology Subcommittee of the Chinese Medical Association in 2015 (17).

Inclusion and exclusion criteria. The diagnostic criteria for preeclampsia were as follows: Normal blood pressure prior to pregnancy with increases at least twice $(\geq 140 / 90 \mathrm{mmHg}$ at an interval of $>4 \mathrm{~h}$ ) after 20 weeks of pregnancy, urinary protein $\geq 300 \mathrm{mg} / 24 \mathrm{~h}$ or proteinuria/creatinine $\geq 0.3 \mathrm{mg} / \mathrm{dl}$, or urine regular protein $2^{+}$and above in random samples. Normal blood pressure prior to pregnancy was defined as normal blood pressure within 1 year before pregnancy without the history of hypertension.

As the group of normal pregnant females, those in late singleton pregnancy with no cardiovascular diseases, renal diseases, endocrine diseases or other chronic diseases complicating the pregnancy or tumors or other complications were enrolled. The exclusion criteria for this group were as follows: Participants with $<28$ weeks of pregnancy, gestational diabetes mellitus, pregnancy complicated with chronic hypertension, peripheral vascular disease history, genital malformation, tumor or any autoimmune diseases.

The subgroup classification of patients with preeclampsia was according to the following standards: Patients with SPE in conformity with the diagnostic criteria of preeclampsia, with blood pressure $\geq 160 / 110 \mathrm{mmHg}$ or PLT $<100 \times 10^{9} / 1$ or serum ALT elevated more than twice the normal limit or serum creatinine twice the normal limit and without other renal diseases. Patients with MPE met the diagnostic criteria for preeclampsia but did not meet the diagnostic criteria for SPE. Early-onset preeclampsia was definitive with the manifestation of preeclampsia prior to 34 weeks of pregnancy. Late-onset preeclampsia was definitive with the manifestation of preeclampsia after 34 weeks of pregnancy. Patients with HELLP syndrome had at least one of the following abnormal findings: Intravascular hemolysis, PLT $<100 \times 10^{9} / 1$, aspartate aminotransferase $(\mathrm{AST}) \geq 70 \mathrm{U} / 1$, lactate dehydrogenase $(\mathrm{LDH}) \geq 600 \mathrm{U} / 1$.

Data collection and follow-up. The data of the included subjects were recorded after introduction into the program, including parameters such as age, body height and body weight prior to pregnancy, systolic and diastolic blood pressure and whether assisted reproductive technology was employed during pregnancy. In the morning of the 2 nd day after enrollment, $8 \mathrm{ml}$ fasting venous blood from the elbows of the subjects was withdrawn and sub-packaged into EDTA-K2 anticoagulant vacuum tubes. The PLT of $1 \mathrm{ml}$ blood sample was measured within $1 \mathrm{~h}$ and the remaining blood was centrifuged at $769 \times \mathrm{g}$ at $4^{\circ} \mathrm{C}$ for $3 \mathrm{~min}$. The supernatant was filled in fresh centrifuge tubes and stored in the refrigerator at $-80^{\circ} \mathrm{C}$ for determination of AST, ALT, LDH and the extraction of the total RNA content. PLT reagent was acquired from the BioRoYee Co. (cat. no. DA0156) and ELISAs were adopted to test the concentrations of LDH (Human LDH ELISA kit; cat. no. 48T-QS40354; Gersion Bio-Technology Co., Ltd.), AST (Human AST ELISA kit; cat. no. 48T-QS41359; Gersion Bio-Technology Co., Ltd.) and ALT (Human ALT/GPT ELISA kit; cat. no. 48T-QS40443; Gersion Bio-Technology Co., Ltd.) in the serum. Urinary protein test kits (Wanlei Bio Co.) were used to determine the protein content of the urine samples.

The patients were followed up until the time-point of delivery and the number of gestational weeks was recorded. Furthermore, the maternal and fetal outcomes were acquired and recorded after delivery. Adverse pregnancy outcomes were defined as those occurring during follow-up or delivery and included placental abruption, cardiac insufficiency, acute renal injury, cerebrovascular accident, disseminated intravascular coagulation, premature delivery, postpartum hemorrhage, small fetal size for gestational age (SGA), neonatal asphyxia and fetal death. SGA was defined as a birth weight $10 \%$ below 
Table I. Sequences of primers used for PCR.

\begin{tabular}{lcll}
\hline Gene & GenBank accession no. & Forward (5'-3') & Reverse $\left(5^{\prime}-3^{\prime}\right)$ \\
\hline $\begin{array}{l}\text { LncRNA TCL6 } \\
\beta \text {-actin }\end{array}$ & AF195821 & GCTGTCTAAGGGCTCATC & GGAGAAAGGCAAAGAACA \\
\hline
\end{tabular}

lncRNA, long non-coding RNA; TCL6, T-cell leukemia/lymphoma 6.

the normal weight for the same gestational age. Premature infants were defined as those whose gestational age was $<37$ weeks prior to delivery. Neonatal asphyxia was diagnosed if the 1-minute Apgar score of the fetus was $\leq 7$.

Reverse transcription-quantitative PCR (RT-qPCR). The serum samples were preserved in 1.5-ml RNA-free centrifuge tubes centrifuge tubes in the $-80^{\circ} \mathrm{C}$ refrigerator. The concentration of the RNAs was detected within one week. A $0.5-\mathrm{ml}$ serum sample was incubated with TRIzol reagent (Thermo Fisher Scientific, Inc.) at room temperature for $5 \mathrm{~min}$, followed by RNA extraction with $0.2 \mathrm{ml}$ chloroform and centrifugation at $12,000 \mathrm{x} \mathrm{g}$ for $5 \mathrm{~min}$. Subsequently, the total RNA content was purified using the mirVana PARIS kit (cat. no. AM1556; Thermo Fisher Scientific, Inc.) in strict accordance with the manufacturer's protocol. The samples were supplemented with ethanol and passed through a filter cartridge containing a glass-fiber filter to immobilize the RNA content. The filter was then repeatedly rinsed and the RNA content was eluted with a low ionic-strength solution. The concentration and purity of the RNA was determined using an ultraviolet spectrophotometer. The PrimeScript RT reagent kit (Takara Bio, Inc.) was employed to synthesize complementary DNA according to the manufacturer's protocol. ChamQ ${ }^{\mathrm{TM}}$ SYBR qRT-PCR MasterMix (Vazyme Biotech) was used for qPCR according to the manufacturer's protocol. The reaction system was as follows: Initial denaturation of $95^{\circ} \mathrm{C}$ for $10 \mathrm{~min}$, followed by $95^{\circ} \mathrm{C}$ for $10 \mathrm{sec}$ and $60^{\circ} \mathrm{C}$ for $60 \mathrm{sec}$ for a total of $45 \mathrm{cycles}$. The $2^{-\triangle \Delta \mathrm{Cq}}$ method (18) was adopted to estimate the relative expression of TCL6 with standardization to $\beta$-actin as the internal control. The primer sequences are listed in Table I.

Statistical analysis. SPSS 21.0 statistical software (IBM Corp.) and GraphPad Prism 6.0 software (GraphPad Software Inc.) were used to analyze data and plotting of graphs. The Shapiro-Wilk test was used to analyze the data for normality of distribution. Continuous variables with a normal distribution are expressed as the mean \pm standard deviation. An independent-samples t-test was used for comparison between two groups. Measurement data with a non-normal distribution were expressed as the mean (interquartile range) and the Mann-Whitney U-test was used for comparison between groups. Enumeration data were expressed as $\mathrm{n}(\%)$ and comparisons between groups were performed with the $\chi^{2}$ test. Receiver operating characteristic (ROC) curve analysis was used to evaluate the diagnostic efficacy of parameters and determine the cut-off values. The $\chi^{2}$ test and Kaplan-Meier curves with the log-rank test were used to analyze the effect of the expression of IncRNA TCL6 on adverse pregnancy.
Logistic regression model was used to evaluate the influencing factors of adverse pregnancy. Single-factor analysis of each influencing factor was performed, the factors with $\mathrm{P}<0.1$ were included in the multivariate logistic regression analysis and for the independent variable screening method, the Enter method provided by SPSS was selected. The P-values calculated for all comparisons were two-sided and $\mathrm{P}<0.05$ was considered to indicate statistical significance.

\section{Results}

Clinical baseline characteristics of included subjects. A total of 100 pregnant women were included in the normal group with an age of 27.0 years (range, 24.7-28.8 years) and 120 pregnant women were included in the preeclampsia group with an age of 25.9 years (range, 25.0-28.1 years). No significant differences were evident in parameters such as age, pre-pregnancy body mass index and PLT between patients with preeclampsia and normal controls $(\mathrm{P}>0.05)$. In comparison with the normal pregnant females, the systolic pressure, diastolic pressure, serum AST, ALT and LDH levels as well as 24-h urinary protein of patients with preeclampsia were higher. The proportion of pregnant females who used assisted reproductive technology for their pregnancy was higher relative to that of pregnant females in the control group $(\mathrm{P}<0.05$; Table II).

LncRNA TCL6 is upregulated in the blood of patients with preeclampsia. The expression pattern of IncRNA TCL6 in the serum of the pregnant women between the normal pregnant females and patients with preeclampsia was compared in the present study. The results suggested that the expression level of TCL6 in the normal pregnant females relative to $\beta$-actin was $1.003 \pm 0.076$, while that in the preeclampsia group was $1.709 \pm 0.439$. The expression of TCL6 in the preeclampsia group was significantly higher compared with that in the normal control group ( $\mathrm{P}<0.01$; Fig. 1A). Furthermore, the ROC curve was plotted for TCL6 expression to distinguish between patients with preeclampsia and normal pregnant females (Fig. 1B). The results suggested that the area under the curve (AUC) was 0.9468 and the cutoff value was 1.206 (the sensitivity was $86.7 \%$ and the specificity was $100 \%$ ). These results indicated that a serum level of lncRNA TCL6 of $>1.206$ relative to $\beta$-actin is able to identify patients with preeclampsia, which may aid the diagnosis of the condition.

LncRNA TCL6 is a biomarker for grading the severity of preeclampsia. To investigate the involvement of 1ncRNA TCL6 in preeclampsia, the patients with preeclampsia were stratified into the following subgroups: SPE and MPE, early-onset 
Table II. Comparison of clinical baseline characteristics.

\begin{tabular}{lcccc}
\hline Characteristic & Normal value & Normal group $(\mathrm{n}=100)$ & Preeclampsia group $(\mathrm{n}=120)$ & P-value \\
\hline Baseline maternal age (years) & - & $27.0(24.7-28.8)$ & $25.9(25.0-28.1)$ & 0.460 \\
Gestational age at baseline (weeks) & - & $29.1(28.1-30.2)$ & $29.2(28.0 \pm 30.5)$ & $38.5(35.1-40.3)$ \\
Gestational age at delivery (weeks) & $37-41$ & $37.8(36.0-40.6)$ & $23.6(21.5-26.0)$ & 0.152 \\
Pre-pregnancy BMI $\left(\mathrm{kg} / \mathrm{m}^{2}\right)$ & - & $24.7(21.1-28.1)$ & $154.6(147.7-160.1)$ & 0.271 \\
Systolic blood pressure $(\mathrm{mmHg})$ & $90-140$ & $118.9(108.3-128.5)$ & $101.9(97.0-105.8)$ & $<0.001$ \\
Diastolic blood pressure $(\mathrm{mmHg})$ & $60-90$ & $76.6(68.5-83.5)$ & $65.9(35.2-107.7)$ & $<0.001$ \\
AST (U/1) & $13-35$ & $26.7(19.4-32.4)$ & $42.2(20.5-80.3)$ & $<0.001$ \\
ALT (U/l) & $7-40$ & $15.1(10.1-18.9)$ & $200.5(218.4-235.9)$ & 0.070 \\
LDH (U/l) & $100-240$ & $154.7(106.9-191.5)$ & $2.41(1.41-4.57)$ & $41(34.2)$
\end{tabular}

Enumeration data are expressed as $\mathrm{n}(\%)$ and the $\chi^{2}$ test was used for comparison between groups. Continuous variables are expressed as the median (interquartile range) and the Mann-Whitney U-test was used for comparison between groups. AST, aspartate aminotransferase; ALT, alanine aminotransferase; LDH, lactate dehydrogenase; PLT, platelets.
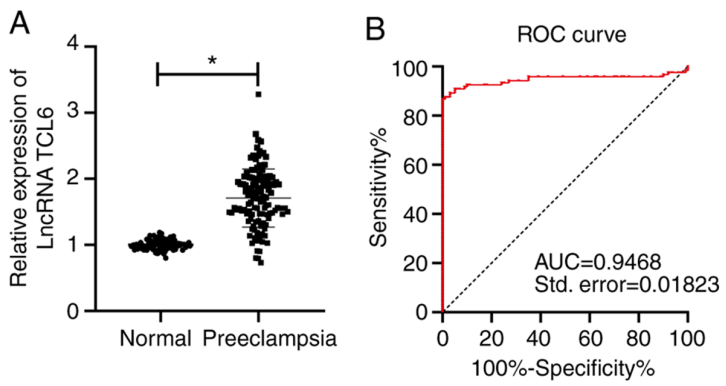

Figure 1. LncRNA TCL6 was upregulated in the blood of patients with preeclampsia. (A) Reverse transcription-quantitative PCR was used to detect the expression of lncRNA TCL6 in serum. (B) ROC curve for the utility of lncRNA TCL6 in the diagnosis of preeclampsia. The independent-samples t-test was used to compare the data in panel A. ${ }^{*} \mathrm{P}<0.05$. IncRNA, long non-coding RNA; TCL6, T-cell leukemia/lymphoma 6; ROC, receiver operating characteristic; AUC, area under the ROC curve; Std., standard error.

preeclampsia and late-onset preeclampsia, preeclampsia complicated with HELLP syndrome and preeclampsia without the complication of the HELLP syndrome. The classification of the patients with preeclampsia was as follows: The 120 preeclampsia patients included 60 MPE patients and 60 SPE patients, including 63 cases of early-onset preeclampsia, 57 cases of late-onset preeclampsia and 10 cases of HELLP syndrome. The ROC curves were plotted to examine the ability of IncRNA TCL6 to distinguish between the different pairs of groups. The results suggested that the expression of TCL6 in the SPE group was significantly higher than that in the MPE group (Fig. 2A) and the ROC curve for distinguishing between these groups had an AUC of 0.7947 at the cutoff value of 1.838 ( $\mathrm{P}<0.0001$; Fig. 2B), sensitivity of $65 \%$ and specificity of $85 \%$. The expression of TCL6 in patients with early-onset preeclampsia was significantly higher compared with that in patients with late-onset preeclampsia (Fig. 2C) and the ROC curve for distinguishing between these groups had an AUC of 0.7700 at the cutoff value of $1.776(\mathrm{P}<0.0001$; Fig. 2D), sensi-
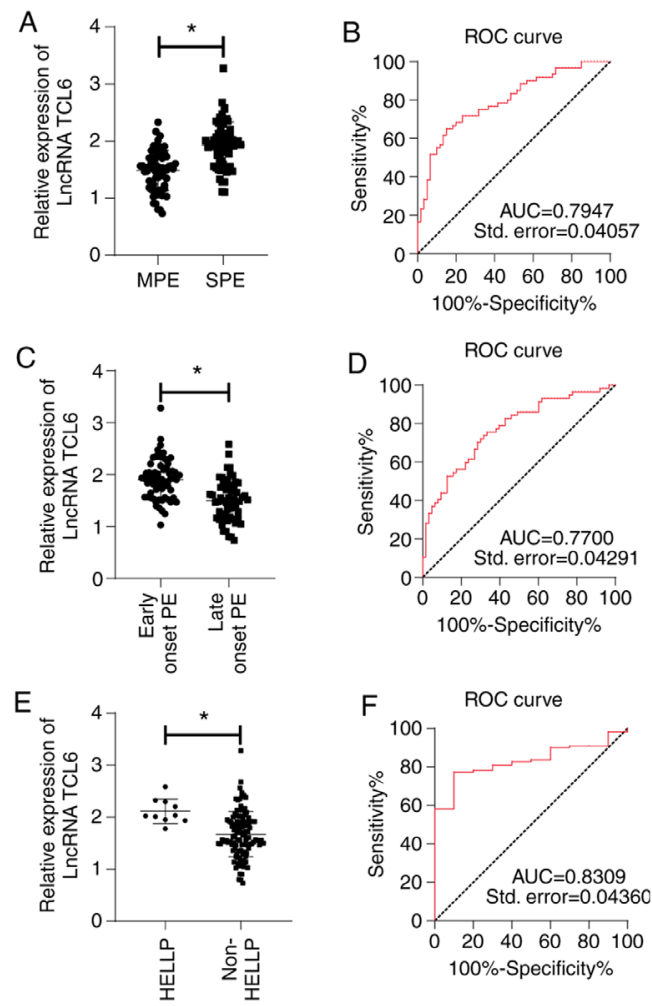

Figure 2. LncRNA TCL6 was upregulated in the blood of each subgroup of patients with preeclampsia. LncRNA TCL6 expression in different subgroups of preeclampsia was detected by reverse transcription-quantitative PCR. (A) Difference in TCL6 expression between SPE and MPE groups. (B) ROC curve of lncRNA TCL6 in the diagnosis of severe preeclampsia. (C) Difference in TCL6 expression between early-onset preeclampsia and late-onset preeclampsia groups. (D) ROC curve of IncRNA TCL6 in the diagnosis of early-onset preeclampsia. (E) Difference in TCL6 expression between patients with preeclampsia with and without HELLP syndrome. (F) ROC curve of lncRNA TCL6 in the diagnosis of HELLP syndrome in patients with preeclampsia. Independent-samples t-tests were used to compare the data in panels $\mathrm{A}, \mathrm{C}$ and $\mathrm{E}$. ${ }^{*} \mathrm{P}<0.05$. IncRNA, long non-coding RNA; TCL6, T-cell leukemia/lymphoma 6; ROC, receiver operating characteristic; AUC, area under the ROC curve; Std., standard error; HELLP, Haemolysis, Elevated Liver enzymes and Low Platelet count; PE, preeclampsia; SPE, severe PE; MPE, mild PE. 
Table III. Comparison of pregnancy outcomes for patients with preeclampsia with different expression of TCL6.

\begin{tabular}{|c|c|c|c|c|}
\hline Expression of TCL6 & Adverse outcomes, n (\%) & Normal delivery, n (\%) & $\mathrm{P}$-value & Total, $\mathrm{n}$ \\
\hline Low $(\geq 1.708)$ & $19(30.2 \%)$ & $41(71.9 \%)$ & $\leq$ & 60 \\
\hline High $(<1.708)$ & $44(69.8 \%)$ & $16(28.1 \%)$ & 0.001 & 60 \\
\hline Total, $\mathrm{n}$ & 63 & 57 & & 120 \\
\hline
\end{tabular}

TCL6, T-cell leukemia/lymphoma 6.

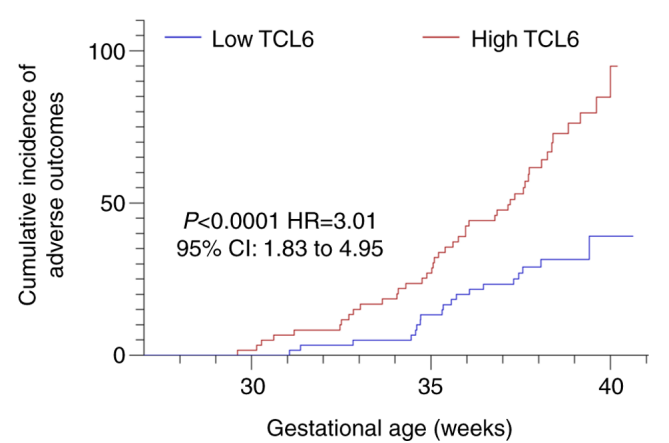

Figure 3. Cumulative incidence of adverse pregnancy outcomes was higher in the high TCL6 expression group. The Kaplan-Meier method was used to analyze the influence of TCL6 levels on pregnancy outcomes in patients with preeclampsia. Compared with that in the low-expression group, the curve in the high-expression group shifted left. TCL6, T-cell leukemia/lymphoma 6; $\mathrm{HR}$, hazard ratio.

tivity of $75.4 \%$ and specificity of $66.7 \%$. Compared with that in the patients with preeclampsia without HELLP syndrome, the level of TCL6 in patients with HELLP syndrome was significantly higher (Fig. 2E) and the ROC curve for distinguishing between these groups had an AUC of 0.8309 at the cutoff value of 1.928 (P<0.001; Fig. 2F), sensitivity of $77.3 \%$ and specificity of $90.0 \%$.

High expression of IncRNA TCL6 increases the risk of adverse pregnancy outcomes. Patients with preeclampsia were allocated to a low expression group and a high expression group according to the median expression of lncRNA TCL6 in this study. Comparison of the incidence of adverse pregnancy outcomes between the two groups indicated that the prognosis of the two groups was significantly different $\left(\chi^{2}=20.89 ; \mathrm{P}<0.0001\right)$. The incidence of adverse pregnancy outcomes in the low expression group was $31.7 \%$, which was lower than that in the high expression group $(73.3 \%$; Table III). Kaplan-Meier analysis indicated a left shift in the curve of the TCL6 high-expression group ( $\mathrm{P}<0.001$; Fig. 3 ), which indicated that at the same gestational week, the cumulative incidence of adverse pregnancy outcomes was higher in the high-expression group than in the low-expression group. These results suggested that the increased expression of TCL6 was associated with adverse pregnancy outcomes.

High expression of IncRNA TCL6 is an independent risk factor for adverse pregnancy outcomes in patients with preeclampsia. Existing evidence has indicated that indexes such as advanced age, overweight or obesity, assisted reproduc-

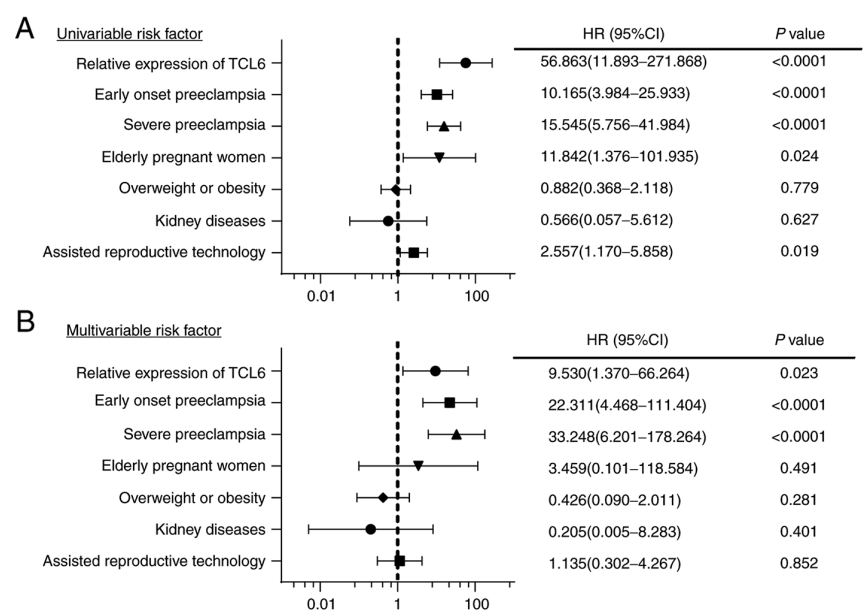

Figure 4. TCL6 expression is an independent risk factor for adverse pregnancy outcomes. (A) Univariate analysis suggested that SPE, early-onset preeclampsia, TCL6 expression, advanced maternal age ( $\geq 35$ years) and assisted reproductive technology were statistically significant risk factors. (B) Multivariate regression analysis indicated that SPE, early-onset preeclampsia and high TCL6 expression were independent risk factors for adverse pregnancy outcomes. In the forest plot, the horizontal lines represent the confidence interval. TCL6, T-cell leukemia/lymphoma 6; SPE, severe preeclampsia; HR, hazard ratio.

tive technology and kidney disease of the mother are high-risk factors of adverse outcomes of singleton pregnancies affected by preeclampsia (19-21). To provide an accurate evaluation of the effect of TCL6 on pregnancy outcomes, SPE, early-onset preeclampsia, TCL6 expression, advanced age, overweight or obesity prior to pregnancy, assisted reproductive technology and kidney disease were included as observational indexes in the regression analysis. As indicated in Fig. 4A, all variables except for overweight/obesity and kidney disease were significant risk factors $(\mathrm{P}<0.1)$. Multivariate logistic regression analysis then indicated that SPE, early-onset preeclampsia and TCL6 expression were independent risk factors for adverse pregnancy outcomes (all $\mathrm{P}<0.05$; Fig. 4B). For each unit increase in the relative expression of TCL6, a 9.5-fold increase in the risk of adverse maternal and fetal outcomes was determined.

\section{Discussion}

Preeclampsia is a common syndrome among pregnant females and is a high risk factor of mortality of pregnant females and their infants (1). According to a previous study, lncRNA TCL6 expression was highly expressed in the placenta of pregnant females with preeclampsia (15). The present study indicated 
that maternal plasma levels of IncRNA TCL6 were increased in patients with preeclampsia, and that determination of TCL6 is supportive in the diagnosis and grading of preeclampsia and it fundamentally serve as an independent risk factor for adverse pregnancy outcomes.

Certain studies have illustrated the importance of blood pressure in the diagnosis and management of preeclampsia (22). $\mathrm{LDH}$ is able to independently function as an indicator for the risk of preeclampsia (23). According to the clinical baseline characteristics of the included subjects, compared with those in normal pregnant females, the alterations in blood pressure, AST, ALT and LDH levels and 24-h urinary protein of patients with preeclampsia were higher. In consistency with this, a study demonstrated the utility of elevated systolic blood pressure and diastolic blood pressure with significant proteinuria after the 20th week of pregnancy as potential factors for the diagnosis of preeclampsia (24). Hence, in the present study, it was speculated that increased blood pressure, serum AST, ALT and LDH levels and 24-h urinary protein may serve as fundamental risk factors of preeclampsia.

Various studies have demonstrated an association between low expression of TCL6 and the occurrence and prognosis of renal hyaluronic cell carcinoma, acute B-lymphocyte leukemia and hepatocarcinoma (10-12). However, the expression and the diagnostic value of TCL6 in preeclampsia remained to be fully determined. The present study indicated that the expression of TCL6 was higher in patients with preeclampsia. The ROC curve demonstrated that the AUC was 0.9468 , while the cutoff value was 1.206 (the sensitivity was $86.7 \%$ and the specificity was $100 \%$ ), thereby indicating that relative serum levels of IncRNA TCL6 >1.206 may aid the diagnosis of preeclampsia. Consistently, dysregulation of a variety of lncRNAs, such as IncRNAs MEG3, IGF2/H19, HOTAIR, SPRY4-IT1 and MALAT1, has been evident in the placenta tissues of pregnant females with preeclampsia (25). In the present study, IncRNA TCL6 was upregulated in the blood of pregnant patients with preeclampsia and detection of high expression of lncRNA TCL6 was indicated to be able to contribute to the diagnosis of preeclampsia.

Research has also focused on the concepts of early-onset preeclampsia and late-onset preeclampsia (6). In the present study, differences in IncRNA TCL6 expression between subgroups of patients with preeclampsia according to time of onset and severity were examined. The results revealed that the expression of TCL6 in the SPE group was higher than the expression in the MPE group, thereby indicating that TCL6 may serve as a diagnostic marker for the severity of preeclampsia. TCL6 levels in patients with early-onset preeclampsia were also higher than those in patients with late-onset preeclampsia, indicating the utility of TCL6 as a marker for the onset time of preeclampsia. In comparison with that in patients with preeclampsia without HELLP syndrome, TCL6 was significantly increased in patients with preeclampsia with HELLP syndrome, indicating the functionality of TCL6 as a factor to diagnose the preeclampsia complication HELLP syndrome. A previous study reported that the microcirculatory flow index and perfused vessel density of pregnant females with preeclampsia complicated with the HELLP syndrome were lower than those of the patients with preeclampsia without HELLP syndrome, while the heterogeneity index of pregnant females with preeclampsia complicated with HELLP syndrome was higher than the index of patients with preeclampsia without HELLP syndrome, thereby indicating that patients with HELLP syndrome may have a worse prognosis (26). To conclude, IncRNA TCL6 may be adopted as a biomarker for grading the severity of preeclampsia. As a prospective study, the present study determined the expression pattern of TCL6 in the serum of patients with preeclampsia for the first time and explored the role of TCL6 expression in the diagnosis and classification of preeclampsia by ROC curve analysis.

The diagnosis of preeclampsia poses an associated risk of developing maternal complications leading to adverse perinatal outcomes (27). To identify the role of TCL6 expression in pregnancy outcomes, pregnant females with preeclampsia were stratified into low and high TCL6 expression groups in the present study. The results were indicative of a lower incidence of adverse pregnancy outcomes in the low-expression group relative to the high-expression group. Previous studies have indicated the association of factors such as obesity, assisted reproductive technology, kidney disease with adverse pregnancy outcomes (19-21). Early-onset preeclampsia conferred a high risk of fetal death relative to late-onset preeclampsia (28). To accurately evaluate the effect of TCL6 on pregnancy outcomes, factors such as SPE, early onset of preeclampsia, TCL6 expression, advanced maternal age, overweight or obesity prior to pregnancy, assisted reproductive technology and kidney disease were included as observation indexes in the regression analysis. The results indicated that SPE, early-onset preeclampsia and TCL6 expression were independent risk factors for adverse pregnancy outcomes. It was determined that for each unit increase in TCL6 expression, the risk of adverse maternal and fetal outcomes increased by 9.5 -fold.

TCL6 was initially flagged as a candidate gene with potential involvement in leukemia (9). Studies have illustrated the participation of TCL6 in clear-cell renal cell carcinoma, breast cancer, retinoblastoma, liver cirrhosis and threatened abortion $(10,13,14,29,30)$. The present results suggested that high expression of 1ncRNA TCL6 was associated with the pathogenesis, severity and poor prognosis of cases of preeclampsia and it may be worthwhile to explore the underlying mechanisms. Following extensive investigation of the pathogenesis of preeclampsia, Redman (31) proposed the "six stages' of preeclampsia in 2014: The first stage ranges from fertilization to embryo implantation, which is a relatively short process. The second stage is $8-18$ weeks of gestation, during which the trophoblast initiates invasion into the uterine spiral artery, which is the vital aspect of placentation. The third stage is a stress response after abnormal placental formation. The fourth stage is the second half of pregnancy, where various types of placental injury factors are released into the maternal blood circulation. In the fifth stage, patients exhibit the clinical manifestations of rising blood pressure. The sixth stage is the period of acute aggravation of the disease, during which atherosclerosis or thrombosis of the spiral artery may occur, and placenta infarction may also take place. Pathophysiological alterations in trophoblast cells are vital for the pathogenesis of preeclampsia. Previous research has validated the involvement of lncRNAs in the proliferation, migration, invasion, apoptosis and other biological functions 
of trophoblasts. In the present study, it was speculated that IncRNA TCL6 may have a pathogenic role by regulating the biological behaviors of trophoblast cells. In addition, in light of the similarity between the biological behavior of placental trophoblast and tumor cells, a study has indicated that TCL6 is able to inhibit the proliferation, migration and invasion of hepatoma cells (12). Therefore, TCL6 may induce abnormal biological behaviors of trophoblast cells, including proliferation, migration and invasion. The study of $\mathrm{Wu}$ et al (15) validated the ability of overexpression of 1ncRNA TCL6 to inhibit the proliferation of trophoblast cells, thus highlighting its involvement in the pathogenesis of preeclampsia. The study of Liu and Gong (14) reported that IncRNA-TCL6 inhibited the proliferation of trophoblast cells by regulating the EGFR pathway in vitro. Considering the significance of the EGFR pathway in the proliferation and differentiation of trophoblast cells and placental development, it was speculated in the present study whether IncRNA TCL6 serves as a regulator of the biological behaviors of trophoblast cells by affecting the EGFR pathway, thus affecting the occurrence of preeclampsia, which requires to be assessed in further studies.

To conclude, the present study supported that high expression of lncRNA TCL6 may assist the diagnosis and grading of preeclampsia and may be functionally utilized as an independent risk factor for adverse pregnancy outcomes. Since the time of sample collection in the present study was from the third trimester of pregnancy to delivery, the determination of TCL6 levels may have been affected. As the sample size in the present study was small, further research with a larger sample size is necessary and a multi-center study should be performed to further clarify the diagnostic and prognostic value of TCL6. In addition, determination of the serum levels of TCL6 in the first or second trimester of pregnancy should be considered to investigate its diagnostic and prognostic value in the early stage of the disease.

\section{Acknowledgements}

Not applicable.

\section{Funding}

No funding was received.

\section{Availability of data and materials}

The datasets used and/or analyzed during the current study are available from the corresponding author on reasonable request.

\section{Authors' contributions}

HW and HM confirm the authenticity of all the raw data. HW and HM contributed to the study concepts, study design, definition of intellectual content, literature research, manuscript preparation, manuscript editing and manuscript review. HW, GS, ML, LM and HM contributed to the clinical study, performing experiments and data acquisition. HW, GS and HM contributed to data analysis and statistical analysis. All authors have read and approved the final manuscript.

\section{Ethics approval and consent to participate}

The experiments were authorized by the academic ethics committee of the Second People's Hospital of Yunnan Province (Kunming, China; no. 2020040). All procedures were strictly implemented according to the Declaration of Helsinki. All the subjects involved were fully informed of the objective of the study and they consented to being enrolled in the study and provided written informed consent prior to sampling.

\section{Patient consent for publication}

Not applicable.

\section{Competing interests}

The authors declare that they have no competing interests.

\section{References}

1. Bokslag A, van Weissenbruch M, Mol BW and de Groot CJ: Preeclampsia; short and long-term consequences for mother and neonate. Early Hum Dev 102: 47-50, 2016.

2. Pauli JM and Repke JT: Preeclampsia: Short-term and Long-term Implications. Obstet Gynecol Clin North Am 42: 299-313, 2015.

3. Ahmed A, Rezai H and Broadway-Stringer S: Evidence-Based Revised View of the Pathophysiology of Preeclampsia. Adv Exp Med Biol 956: 355-374, 2017.

4. Balogun OA and Sibai BM: Counseling, Management, and Outcome in Women With Severe Preeclampsia at 23 to 28 Weeks Gestation. Clin Obstet Gynecol 60: 183-189, 2017.

5. Aloizos S, Seretis C, Liakos N, Aravosita P, Mystakelli C, Kanna E and Gourgiotis S: HELLP syndrome: Understanding and management of a pregnancy-specific disease. J Obstet Gynaecol 33: 331-337, 2013

6. Sones JL and Davisson RL: Preeclampsia, of mice and women. Physiol Genomics 48: 565-572, 2016.

7. Nagy B: Cell-free nucleic acids in prenatal diagnosis and pregnancy-associated diseases. EJIFCC 30: 215-223, 2019.

8. McAninch D, Roberts CT and Bianco-Miotto T: Mechanistic Insight into Long Noncoding RNAs and the Placenta. Int J Mol Sci 18: 18, 2017.

9. Saitou M, Sugimoto J, Hatakeyama T, Russo G and Isobe M: Identification of the TCL6 genes within the breakpoint cluster region on chromosome 14q32 in T-cell leukemia. Oncogene 19: 2796-2802, 2000.

10. Chen Z, Zhuang Q, Cheng K, Ming Y, Zhao Y, Ye Q and Zhang S: Long non-coding RNA TCL6 enhances preferential toxicity of paclitaxel to renal cell carcinoma cells. J Cancer 11: 1383-1392, 2020.

11. Cuadros M, Andrades Á, Coira IF, Baliñas C, Rodríguez MI, Álvarez-Pérez JC, Peinado P, Arenas AM, García DJ, Jiménez P, et al: Expression of the long non-coding RNA TCL6 is associated with clinical outcome in pediatric B-cell acute lymphoblastic leukemia. Blood Cancer J 9: 93, 2019.

12. Luo LH, Jin M, Wang LQ, Xu GJ, Lin ZY, Yu DD, Yang SL, Ran RZ, Wu G and Zhang T: Long noncoding RNA TCL6 binds to miR-106a-5p to regulate hepatocellular carcinoma cells through PI3K/AKT signaling pathway. J Cell Physiol 235: 6154-6166, 2020.

13. Zhang Y,LiZ, Chen M, Chen H,Zhong Q, Liang L and LiB: IncRNA TCL6 correlates with immune cell infiltration and indicates worse survival in breast cancer. Breast Cancer 27: 573-585, 2020.

14. Liu LP and Gong YB: LncRNA-TCL6 promotes early abortion and inhibits placenta implantation via the EGFR pathway. Eur Rev Med Pharmacol Sci 22: 7105-7112, 2018.

15. Wu JL, Wang YG, Gao GM, Feng L, Guo N and Zhang CX: Overexpression of 1ncRNA TCL6 promotes preeclampsia progression by regulating PTEN. Eur Rev Med Pharmacol Sci 23: 4066-4072, 2019.

16. No authors listed: Hypertension in pregnancy. Report of the American College of Obstetricians and Gynecologists' Task Force on Hypertension in Pregnancy. Obstet Gynecol 122: 1122-1131, 2013. 
17. Hypertensive Disorders in Pregnancy Subgroup, Chinese Society of Obstetrics and Gynecology, Chinese Medical Association and Hypertensive Disorders in Pregnancy Subgroup Chinese Society of Obstetrics and Gynecology Chinese Medical Association: Diagnosis and treatment guideline of hypertensive disorders in pregnancy. Zhonghua Fu Chan Ke Za Zhi 50: 721-728, 2015 (In Chinese)

18. Schmittgen TD and Livak KJ: Analyzing real-time PCR data by the comparative C(T) method. Nat Protoc 3: 1101-1108, 2008.

19. Hui D and Hladunewich MA: Chronic Kidney Disease and Pregnancy. Obstet Gynecol 133: 1182-1194, 2019.

20. Marsh CA and Hecker E: Maternal obesity and adverse reproductive outcomes: Reducing the risk. Obstet Gynecol Surv 69: 622-628, 2014

21. Reddy UM, Wapner RJ, Rebar RW and Tasca RJ: Infertility, assisted reproductive technology, and adverse pregnancy outcomes: Executive summary of a National Institute of Child Health and Human Development workshop. Obstet Gynecol 109: 967-977, 2007.

22. Ashworth DC, Maule SP, Stewart F, Nathan HL, Shennan AH and Chappell LC: Setting and techniques for monitoring blood pressure during pregnancy. Cochrane Database Syst Rev 8: CD012739, 2020.

23. Han Q, Zheng W, Guo XD, Zhang D, Liu HF, Yu L and Yan JY: A new predicting model of preeclampsia based on peripheral blood test value. Eur Rev Med Pharmacol Sci 24: 7222-7229, 2020.

24. Morris RK, Riley RD, Doug M, Deeks JJ and Kilby MD: Diagnostic accuracy of spot urinary protein and albumin to creatinine ratios for detection of significant proteinuria or adverse pregnancy outcome in patients with suspected pre-eclampsia: Systematic review and meta-analysis. BMJ 345 (jul09 1): e4342, 2012.
25. Song X,Luo X, Gao Q, Wang Y, Gao Q and Long W: Dysregulation of LncRNAs in Placenta and Pathogenesis of Preeclampsia. Curr Drug Targets 18: 1165-1170, 2017.

26. Cornette J, Herzog E, Buijs EA, Duvekot JJ, Rizopoulos D, Hop WC, Tibboel D and Steegers EA: Microcirculation in women with severe pre-eclampsia and HELLP syndrome: A case-control study. BJOG 121: 363-370, 2014.

27. Guida JPS, Surita FG, Parpinelli MA and Costa ML: Preterm Preeclampsia and Timing of Delivery: A Systematic Literature Review. Rev Bras Ginecol Obstet 39: 622-631, 2017.

28. Lisonkova S and Joseph KS: Incidence of preeclampsia: risk factors and outcomes associated with early- versus late-onset disease. Am J Obstet Gynecol 209: 544.e1-544.e12, 2013.

29. Li LJ, Wu XY, Tan SW, Xie ZJ, Pan XM, Pan SW, Bai WR, Li HJ, Liu HL, Jiang J, et al: Lnc-TCL6 is a potential biomarker for early diagnosis and grade in liver-cirrhosis patients. Gastroenterol Rep (Oxf) 7: 434-443, 2019.

30. Tao S, Wang W, Liu P, Wang $\mathrm{H}$ and Chen W: Long non-coding RNA T-cell leukemia/lymphoma 6 serves as a sponge for miR-21 modulating the cell proliferation of retinoblastoma through PTEN. Korean J Physiol Pharmacol 23: 449-458, 2019.

31. Redman C: The six stages of pre-eclampsia. Pregnancy Hypertens 4: 246, 2014.

This work is licensed under a Creative Commons Attribution-NonCommercial-NoDerivatives 4.0 International (CC BY-NC-ND 4.0) License. 